Original Article

\title{
Administration of Corticosteroids, Ascorbic Acid, and Thiamine Improves Oxygenation after Thoracoscopic Esophagectomy
}

\author{
Tadashi Matsuoka, MD, PhD, ${ }^{1}$ Hiroharu Shinozaki, MD, PhD,${ }^{1}$ Soji Ozawa, MD, PhD, ${ }^{2}$ \\ Yoshimitsu Izawa, MD, PhD, ${ }^{1}$ Kazuo Koyanagi, $\mathrm{MD}, \mathrm{PhD},{ }^{2}$ \\ Alan Kawarai Lefor, MD, MPH, PhD, FACS, ${ }^{3}$ and Kenji Kobayashi, MD ${ }^{1}$
}

\begin{abstract}
Purpose: The activity of corticosteroids, ascorbic acid, and thiamine against oxidative and inflammatory responses was evaluated in patients undergoing esophagectomy. This study was undertaken to investigate the effect of this combined therapy on lung dysfunction following esophagectomy.

Methods: In this retrospective before-after study, we compared the clinical course of consecutive patients undergoing thoracoscopic esophagectomy treated with the combination of corticosteroids, ascorbic acid, and thiamine between June and December 2018 with a control group treated with corticosteroids alone between January 2016 and May 2018. Outcomes included oxygenation (arterial partial pressure of oxygen $\left(\mathrm{PaO}_{2}\right) /$ fractional concentration of inspired oxygen $\left(\mathrm{FiO}_{2}\right)$ ratios), duration of mechanical ventilation and intensive care unit (ICU) length of stay.

Results: In all, 17 patients were included in this study (6 in the combination therapy group and 11 patients in the control group). Mean $\mathrm{PaO}_{2} / \mathrm{F}_{\mathrm{i}} \mathrm{O}_{2}$ ratios in the combined therapy group were significantly higher than in the control group at all points during the observation period $(p<0.001)$. In the combined therapy group, the duration of mechanical ventilation and ICU stay were significantly shorter $(p<0.001, p=0.009)$.

Conclusions: This study suggests that combined therapy including corticosteroids, ascorbic acid, and thiamine may be effective in improving oxygenation after esophagectomy. Additional studies are required to confirm these preliminary findings.
\end{abstract}

Keywords: esophagectomy, ascorbic acid, corticosteroids, lung injury

\section{Introduction}

Esophagectomy is an invasive surgical procedure. ${ }^{1)}$ Thoracoscopic esophagectomy is commonly performed resulting in a less invasive operation with less impact on the patient. However, the incidence of perioperative morbidity remains as high as $40 \%$. $^{2,3)}$ Impaired oxygenation, believed to result from surgical stress and one lung ventilation $^{4)}$ is a well-known complication of esophagectomy, and requires treatment ranging from oxygen

${ }^{1}$ Department of Surgery, Saiseikai Utsunomiya Hospital, Utsunomiya, Tochigi, Japan

${ }^{2}$ Department of Gastroenterological Surgery, School of Medicine, Tokai University, Isehara, Kanagawa, Japan

${ }^{3}$ Department of Surgery, Jichi Medical University, Shimotsuke, Tochigi, Japan

Received: July 23, 2019; Accepted: September 12, 2019

Corresponding author: Hiroharu Shinozaki, MD, PhD. Department of Surgery, Saiseikai Utsunomiya Hospital, 911-1 Takebayashi-cho, Utsunomiya, Tochigi 321-0974, Japan

Email: hiroharu_shinozaki@saimiya.com

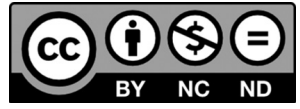

This work is licensed under a Creative Commons Attribution-NonCommercial-

NoDerivatives International License.

(C)2020 The Editorial Committee of Annals of Thoracic and Cardiovascular Surgery 
supplementation to mechanical ventilation. ${ }^{5)}$ Oxidative stress and inflammation are believed to play crucial roles in the pathogenesis of impaired oxygenation.,5) To improve oxygenation, drugs such as elastase inhibitor ${ }^{7)}$ or corticosteroids ${ }^{8,9)}$ are given, but their therapeutic effect has not been confirmed. ${ }^{7,8,10)}$

Combination therapy with corticosteroids, ascorbic acid, and thiamine has a prominent therapeutic effect on oxidative stress and inflammatory response. ${ }^{11,12)}$ These favorable effects can provide significant benefits in various conditions in critically ill patients care such as septic shock ${ }^{13)}$ or acute respiratory distress syndrome. ${ }^{14)}$ It was demonstrated that a synergistic effect of these drugs improves the mortality and morbidity associated with these conditions. ${ }^{11,12)}$ Therefore, we considered that the addition of ascorbic acid and thiamine might confer a further beneficial effect to limit lung dysfunction caused by oxidative stress and inflammation in patients after esophagectomy treated with corticosteroids alone. On the basis of previously reported safety and potential benefits, ${ }^{15)}$ we began to use the combination of intravenous corticosteroids, ascorbic acid, and thiamine as adjunctive therapy for the intraoperative and postoperative management of patients undergoing esophagectomy. We conducted a retrospective before-after pilot study to evaluate impaired oxygenation in patients following esophagectomy comparing patients treated with intravenous corticosteroids, ascorbic acid, and thiamine and patients treated with corticosteroids alone.

\section{Materials and Methods}

\section{Study design}

This is a retrospective study, approved by the Institutional Review Board of Saiseikai Utsunomiya Hospital (No. 2018-14). Consecutive patients undergoing planned thoracoscopic esophagectomy for esophageal carcinoma between July and December 2018 were treated with a combination of corticosteroids, ascorbic acid, and thiamine in addition to standard postoperative management of patients after esophagectomy (corticosteroids were used routinely before this study). ${ }^{16)}$ Patients $<20$ years of age, those who received massive transfusions because of excessive intraoperative bleeding, conversion to open esophagectomy and those with limitations of care were not included in this study. The control group consisted of consecutive patients undergoing planned thoracoscopic esophagectomy between January 2016 and May 2018, using the same inclusion and exclusion criteria as the treatment group. The patients' clinical and demographic data were abstracted from the medical records.

\section{Surgical procedure}

All patients underwent thoracoscopic esophagectomy with lymphadenectomy and reconstruction with a gastric tube. During the intra-thoracic portion of the procedure, patients were placed in the left semi-prone position. Three 12-mm trocars and two 5-mm trocars were inserted. The right lung was deflated and gently displaced to the ventral side. Esophageal mobilization and mediastinal lymph node dissection were performed. After the intra-thoracic procedure, both recurrent laryngeal nerves, the aortic wall, left mediastinal pleura, pericardium, membranous portion of the trachea, and diaphragm were exposed in the surgical field. During the intra-abdominal portion, patients were placed in the supine position, and the mobilized esophagus was delivered through the enlarged hiatus. The stomach was prepared for reconstruction by dividing it at the cardia. This stage was performed through a laparotomy. In the cervical portion of the procedure, the cervical esophagus was mobilized using a collar incision, and the prepared gastric tube was pulled up through one of three routes: anterior sternal, retrosternal, or posterior mediastinal. The procedure was completed with a cervical esophagogastric anastomosis.

\section{Perioperative management}

All operations in this study were performed under general anesthesia with epidural analgesia. For standard prophylaxis against lung dysfunction following esophagectomy, methyl-prednisolone $(125 \mathrm{mg} / \text { body })^{8,17)}$ was given at the beginning of the operation ${ }^{16}$ and every 24 hours after the initial administration for 4 days (including the operative day). Postoperatively, all patients underwent mechanical ventilation and were cared for in the intensive care unit (ICU). In the ICU, all patients were treated according to a conservative, physiologically based fluid and vasopressor strategy, ${ }^{18)}$ and underwent mechanical ventilation with a lung-protective strategy (respiratory settings were initiated at low tidal volumes: $6 \mathrm{~mL} / \mathrm{kg}$, adequate positive end-expiratory pressure $\left(5-10 \mathrm{cmH}_{2} \mathrm{O}\right)$ and fractional concentration of inspired oxygen (30\%-40\%), and were modified as oxygenation changed), ${ }^{19)}$ and limited use of sedation. ${ }^{20)}$ Patients received enteral nutrition via a jejunostomy tube started within 24 hours of surgery. Fluid administration was restricted after achieving of hemodynamic stability without vasopressors depending on oxygenation. After restricting fluids, if oxygenation was not 
adequate, diuretics (furosemide, $40 \mathrm{mg} /$ day) were given. Extubation was managed according to the Japanese protocol for liberation from mechanical ventilation, ${ }^{21)}$ when patients were physiologically stable, the ratio of the arterial partial pressure of oxygen $(\mathrm{mmHg})$ to the fractional concentration of inspired oxygen $\left(\mathrm{P}_{\mathrm{a}} \mathrm{O}_{2} / \mathrm{F}_{\mathrm{i}} \mathrm{O}_{2}\right.$ ratio $)>300$, and rapid shallow breathing index $<80 / \mathrm{min} / \mathrm{L}$, spontaneous breathing trial (SBT) was performed. SBT was performed with $\mathrm{F}_{\mathrm{i}} \mathrm{O}_{2} 30 \%$, continuous positive airway pressure $5 \mathrm{cmH}_{2} \mathrm{O}$ and pressure support $5 \mathrm{cmH}_{2} \mathrm{O}$. If the respiratory status of the patient did not deteriorate after an SBT, the patient was extubated.

\section{Protocol for combined treatment with corticosteroids, ascorbic acid, and thiamine}

Consecutive patients during the study period undergoing esophagectomy were given corticosteroids, ascorbic acid, and thiamine. Methyl-prednisolone was given as the corticosteroid with conventional dosing used for esophagectomy in Japan. ${ }^{17)}$ The protocol for ascorbic acid and thiamine used in this study was previously reported. ${ }^{13)}$ Thiamine was included in this protocol to assist in the metabolism of oxalate, a metabolite of ascorbic acid and may potentially cause kidney injury. ${ }^{22)}$ At the beginning of the operation, methyl-prednisolone (125 mg) was administered intravenously over 30 minutes. At the beginning of the intra-thoracic procedure, ascorbic acid $(1.5 \mathrm{~g})$ and thiamine $(200 \mathrm{mg})$ were administered intravenously over 30 minutes. Methyl-prednisolone was given $125 \mathrm{mg}$ every 24 hours postoperatively for 4 days (including the operative day), ascorbic acid was given $1.5 \mathrm{~g}$ every 6 hours after the initial administration for 4 days or until ICU discharge; thiamine was given $200 \mathrm{mg}$ every 12 hours after the initial administration for 4 days or until ICU discharge.

\section{Outcomes}

The primary outcome of this study was oxygenation based on $\mathrm{P}_{\mathrm{a}} \mathrm{O}_{2} / \mathrm{F}_{\mathrm{i}} \mathrm{O}_{2}$ ratio during the first 2 days after surgery. In extubated patients, the $\mathrm{F}_{\mathrm{i}} \mathrm{O}_{2}$ was estimated from settings of the oxygen delivery system. ${ }^{23)}$ Secondary outcomes were duration of mechanical ventilation and ICU stay, the rate of re-intubation, changes in white blood cell count, and serum concentration of $\mathrm{C}$-reactive protein and postoperative complications.

\section{Statistical analysis}

All data are expressed as the mean \pm standard error of the mean. The changes in $\mathrm{P}_{\mathrm{a}} \mathrm{O}_{2} / \mathrm{F}_{\mathrm{i}} \mathrm{O}_{2}$ ratio, white blood cell count, and serum C-reactive protein were analyzed using a mixed-effects model for repeated measures followed by a t-test for post hoc comparisons. The mixed-effects model for repeated measures included treatment group, time, and the treatment-by-time interaction as factors and a random intercept for each subject. Student's t-test and Fisher's exact test were used to test for differences in the remaining variables between the two groups. The differences were considered significant when the $p$ value was $<0.05$. All of the data were analyzed using SPSS 25.0 statistical software (SPSS Inc., Chicago, IL, USA).

\section{Results}

\section{Patient characteristics}

There were six patients in the combination therapy group and 11 patients in the control group. Baseline characteristics are shown in Table 1, Operative and ICU data are presented in Table 2. There were no significant differences in baseline characteristics between the two groups. There were no significant differences in operative and ICU data between the two groups except for cumulative fluid balance and the use of diuretics in the ICU. In the control group, diuretics were used to decrease cumulative fluid balance to prepare for liberation from the ventilator.

\section{Primary outcome}

Oxygenation

Mean $\mathrm{PaO}_{2} / \mathrm{F}_{\mathrm{i}} \mathrm{O}_{2}$ ratios in the combination therapy group were significantly higher than those in the control group throughout the observation period ( $\mathrm{p}<0.001)$. From the end of the operation, mean $\mathrm{PaO}_{2} / \mathrm{F}_{\mathrm{i}} \mathrm{O}_{2}$ ratios of the combination therapy group exceeded that of the control group (Fig. 1). The duration of mechanical ventilation was significantly shorter in the combination therapy group compared with the control group (combination therapy group vs control group, mean \pm SEM: $2.0 \pm 0.0$ vs $4.2 \pm 0.3$ days, $\mathrm{p}<0.001$ ). The rate of re-intubation in the combination therapy group was comparable to that in the control group (0 of $6(0.0 \%)$ vs 1 of $11(9.1 \%), \mathrm{p}=1.000)$ (Table $3)$. The ICU length of stay was shorter in the combination therapy group ( $3.2 \pm 0.3$ vs $6.9 \pm 0.9$ days, $\mathrm{p}=0.009)$.

\section{Secondary outcomes}

\section{Inflammatory response}

There were no differences in the time course of white blood cell count or C-reactive protein level comparing the combination therapy group and the control group 
Table 1 Patient characteristics

\begin{tabular}{|c|c|c|c|}
\hline & Control $(\mathrm{N}=11)$ & $\begin{array}{l}\text { Combination therapy } \\
\qquad(\mathrm{N}=6)\end{array}$ & $\mathrm{p}$ value \\
\hline Age (years) & $69.7 \pm 2.3$ & $67.7 \pm 1.4$ & 0.542 \\
\hline Body mass index $\left(\mathrm{kg} / \mathrm{m}^{2}\right)$ & $22.0 \pm 0.7$ & $21.1 \pm 1.3$ & 0.268 \\
\hline Male N (\%) & $10(91)$ & $4(67)$ & 0.515 \\
\hline Smoking history & & & 0.600 \\
\hline Never & $4(36)$ & $1(17)$ & \\
\hline Former & $7(64)$ & $5(83)$ & \\
\hline Current & $0(0)$ & $0(0)$ & \\
\hline Pack-years & $31.5 \pm 10.2$ & $31.8 \pm 8.2$ & 0.985 \\
\hline ASA performance status & & & 0.353 \\
\hline$\leqq 2$ & $11(100)$ & $5(83)$ & \\
\hline$\geqq 3$ & $0(0)$ & $1(17)$ & \\
\hline Tumor location & & & 0.304 \\
\hline Ut & $0(0)$ & $0(0.0)$ & \\
\hline Mt & $5(45)$ & $5(83)$ & \\
\hline $\mathrm{Lt}$ & $6(55)$ & $1(17)$ & \\
\hline Tumor stage & & & 0.171 \\
\hline $0 / \mathrm{I}$ & $8(73)$ & $5(83)$ & \\
\hline II & $3(27)$ & $0(0)$ & \\
\hline III & $0(0)$ & $1(17)$ & \\
\hline Preoperative chemotherapy & $7(64)$ & $2(33)$ & 0.335 \\
\hline \multicolumn{4}{|l|}{ Lung function } \\
\hline $\begin{array}{l}\text { Obstructive disorder } \\
\text { (FEV1.0\%<70\%) }\end{array}$ & $1(9.1)$ & $0(0.0)$ & 1.000 \\
\hline Restrictive disorder $(\% \mathrm{VC}<80 \%)$ & $0(0.0)$ & $0(0.0)$ & 1.000 \\
\hline Unknown & $1(9.1)$ & $0(0.0)$ & N.A \\
\hline
\end{tabular}

Values are expressed as the mean \pm standard error of the mean or the number (percent). ASA: American Society of Anesthesiologists; FEV: forced expiratory volume; N.A: not available; Ut: upper; Mt: middle; Lt: lower

(Fig. 2A and 2B). In both groups, the white blood cell count gradually increased over time. Although serum C-reactive protein levels in both groups at the end of surgery were comparable to the baseline values, the $\mathrm{C}$-reactive protein level increased thereafter until the second postoperative day and decreased at the third postoperative day.

\section{Postoperative complications}

The incidence of postoperative complications was similar in the two groups. The rate of anastomotic leakage was $2 / 6(33 \%)$ in the combination therapy group and $3 / 11(27 \%)$ in the control group $(\mathrm{p}=1.000)$. The incidence of acute respiratory distress syndrome, pneumonia, and recurrent nerve palsy, was similar in both groups $(\mathrm{p}=1.000, \mathrm{p}=1.000, \mathrm{p}=0.600$, respectively) (Table 3).

\section{Discussion}

In this study, we found that the perioperative administration of the combination of corticosteroids, ascorbic acid, and thiamine reduces the severity of impaired oxygenation after esophagectomy. Paul et al. reported that this combination was effective in preventing progressive organ dysfunction and in reducing the mortality of patients with septic shock. ${ }^{13)}$ To the best of our knowledge, this is the first report that the combination of corticosteroids, ascorbic acid, and thiamine reduces the severity pulmonary dysfunction after esophagectomy.

Impaired oxygenation after esophagectomy may be related to the systemic inflammatory response syndrome, which is invoked by tissue injury associated with surgery and one lung ventilation via inflammatory responses and oxidative stress. $\left.{ }^{4}\right)$ This impairment is believed to begin intraoperatively and continue for several days. ${ }^{24)}$ Even after minimally invasive esophagectomy, which is less invasive than open esophagectomy, impaired oxygenation occurs and necessitates supportive care. ${ }^{2)}$ About $10 \%$ of patients who undergo thoracoscopic esophagectomy need prolonged mechanical ventilation and $7 \%$ of patients underwent unplanned intubation. ${ }^{2)}$ In the present study, the mean $\mathrm{P}_{\mathrm{a}} \mathrm{O}_{2} / \mathrm{F}_{\mathrm{i}} \mathrm{O}_{2}$ ratios in patients who received combination therapy are significantly higher than the control 
Table 2 Operative and intensive care unit data

\begin{tabular}{lccc}
\hline & Control (N=11) & $\begin{array}{c}\text { Combination therapy } \\
(\mathrm{N}=6)\end{array}$ & p value \\
\hline Operating time (min) & $401 \pm 14$ & $407 \pm 22$ & 0.826 \\
Duration of one lung ventilation (min) & $209 \pm 12$ & $215 \pm 12$ & 0.739 \\
Intraoperative blood loss & $335 \pm 95$ & $546 \pm 258$ & 0.366 \\
Reconstruction route & & & 0.301 \\
$\quad$ Anterior sternal & $10(0)$ & $1(17)$ & \\
$\quad$ Retrosternal & $1(9)$ & $5(83)$ & \\
$\quad$ Posterior mediastinal & $65.8 \pm 4.5$ & $0(0)$ & 0.667 \\
Intraoperative fluid given/weight & $50.3 \pm 4.0$ & $54.2 \pm 8.5$ & 0.636 \\
Intraoperative cumulative fluid balance/weight & & & \\
Cumulative fluid balance/weight & $82.1 \pm 5.2$ & $121.7 \pm 17.8$ & 0.013 \\
$\quad$ Postoperative day 1 & $87.0 \pm 9.2$ & $137.5 \pm 29.4$ & 0.049 \\
$\quad$ Postoperative day 2 & $2(18)$ & $2(33)$ & 0.584 \\
Medications given in the intensive care unit & $7(64)$ & $0(0)$ & 0.035 \\
$\quad$ Vasopressors & & & \\
$\quad$ Diuretics &
\end{tabular}

Values are expressed as the mean \pm standard error of the mean or the number (percent).

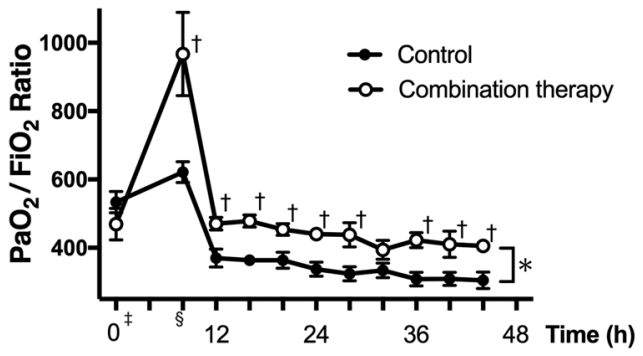

Fig. 1 Changes in $\mathrm{P}_{\mathrm{a}} \mathrm{O}_{2} / \mathrm{F}_{\mathrm{i}} \mathrm{O}_{2}$ ratios. The $\mathrm{P}_{\mathrm{a}} \mathrm{O}_{2} /$ $\mathrm{F}_{\mathrm{i}} \mathrm{O}_{2}$ ratios were recorded from the beginning of surgery to 48 hours postoperatively. $\neq: \mathrm{P}_{\mathrm{a}} \mathrm{O}_{2} / \mathrm{F}_{\mathrm{i}} \mathrm{O}_{2}$ ratios recorded after intubation. §: $\mathrm{P}_{\mathrm{a}} \mathrm{O}_{2} / \mathrm{F}_{\mathrm{i}} \mathrm{O}_{2}$ ratios recorded at the end of operation (almost 8 hours after the beginning). The error bars show the standard error of the mean. * $\mathrm{p}<0.05$ indicates a statistically significant difference using a mixed-effects model for repeated measures. $\uparrow \mathrm{p}<0.05$ compared with the control group as determined by post-hoc analysis and Student's t-test.

group throughout the observation period and all patients in the combined therapy group were extubated on the first postoperative day without subsequent re-intubation. The combination of corticosteroids, ascorbic acid, and thiamine appears to be effective in reducing the severity of lung dysfunction after esophagectomy.

Although ascorbic acid and corticosteroids have antioxidant and anti-inflammatory effects, respectively, it was suggested that the combination of these drugs is essential to produce a significant therapeutic effect. ${ }^{25-28)}$ It was reported that the efficacy of these drugs was seen in patients with
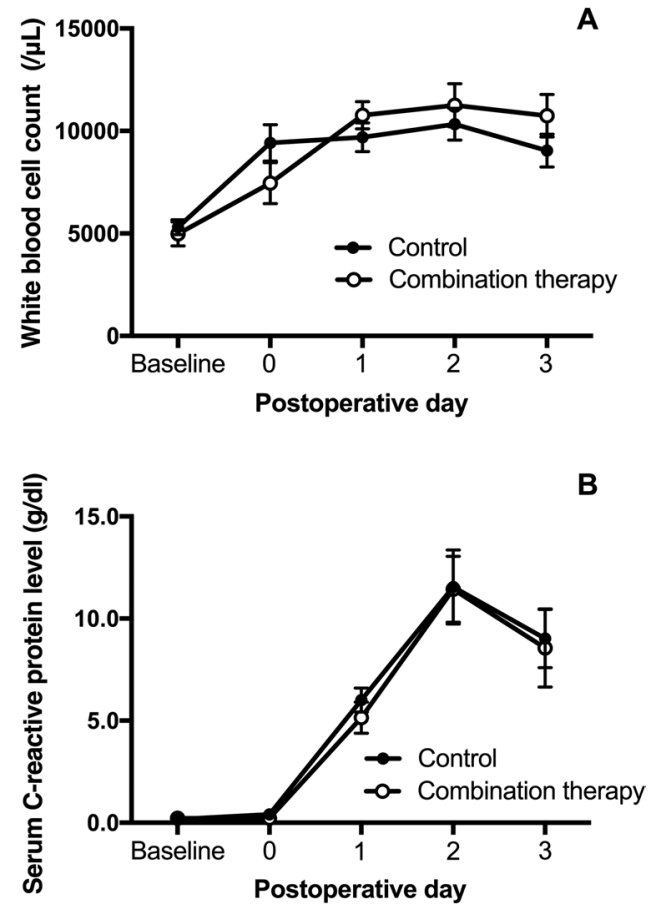

Fig. 2 Changes in white blood cell count (A) and serum C-reactive protein levels (B). White blood cell count and C-reactive protein levels were recorded from the day before surgery as a baseline through the third postoperative day. The error bars show the standard error of the mean.

acute respiratory distress syndrome. ${ }^{14)}$ These drugs are believed to prevent and repair pulmonary endothelial barrier dysfunction or attenuate ischemia/reperfusion injury. ${ }^{12,29)}$ In patients undergoing esophagectomy, we 
Table 3 Outcomes

\begin{tabular}{lccc}
\hline & Control $(\mathrm{N}=11)$ & $\begin{array}{c}\text { Combination therapy } \\
(\mathrm{N}=6)\end{array}$ & p value \\
\hline Mechanical ventilation (days) & $4.2 \pm 0.3$ & $2.0 \pm 0.0$ & $<0.001$ \\
Re-intubation & $1(9)$ & $0(0)$ & 1.000 \\
Tracheotomy & $1(9)$ & $0(0)$ & 1.000 \\
Intensive care unit length of & $6.9 \pm 0.9$ & $3.2 \pm 0.3$ & 0.009 \\
stay (days) & & & \\
Postoperative complications & $1(9)$ & $0(0)$ & 1.000 \\
$\quad$ Acute respiratory distress & $3(27)$ & $2(33)$ & 1.000 \\
$\quad$ syndrome & $5(45)$ & $2(33)$ & 1.000 \\
$\quad \begin{array}{l}\text { Anastomotic leakage } \\
\text { Pneumonia }\end{array}$ & $4(36)$ & $1(17)$ & 0.600 \\
$\quad$ Recurrent nerve palsy & & & \\
\hline
\end{tabular}

The values are expressed as the mean \pm standard error of the mean or the number (percent).

speculated that a similar mechanism is in effect to reduce surgically induced stress, resulting in improved oxygenation. We suggest that the combined treatment given acts at the level of pulmonary endothelial cells, ${ }^{12}{ }^{2}$ and does not result in elevation of the serum $\mathrm{C}$-reactive protein level.

Cumulative fluid balance was significantly higher in the combined therapy group, which may be related to the use of diuretics and restricted fluid administration in the control group. To decrease cumulative fluid balance to improve oxygenation, diuretics were given to patients in the control group. Since the $\mathrm{P}_{\mathrm{a}} \mathrm{O}_{2} / \mathrm{F}_{\mathrm{i}} \mathrm{O}_{2}$ ratios in the combination therapy group were maintained postoperatively, the combined therapy group did not need diuretics and restricted fluid management to improve oxygenation. Excessive diuretic use and restricted fluid management may lead to renal dysfunction due to dehydration. ${ }^{30}$ Diuretics also have a risk of inducing hypokalemia due to potassium excretion and sometimes, hyperkalemia due to excessive replacement. Avoiding the need for diuretics can avoid these risks in the postoperative period.

The safety of administering high-dose ascorbic acid has been reported. ${ }^{22)}$ High amounts of serum oxalate, a metabolite of ascorbic acid and normally excreted by the kidney, might have a risk for tissue deposition and crystallization in the kidney of patients with renal dysfunction if excessive amounts of ascorbic acid are given. ${ }^{22}$ Thiamine helps oxalate metabolism, and a thiamine deficiency increases the conversion of glyoxylate to oxalate. ${ }^{22)}$ Therefore, thiamine was included along with high-dose ascorbic acid and corticosteroid combination therapy in these patients. The dose of ascorbic acid given was less than that in previous reports and did not cause adverse effects. ${ }^{13)}$
This study has acknowledged limitations. It is a small, retrospective study and the two groups were not treated concurrently. This could have affected details of treatment, which could influence the outcomes. The results of this pilot study serve to support the conduct of a larger study, and possibly a randomized prospective trial in the future. The addition of ascorbic acid is associated with low risk at low cost.

\section{Conclusion}

The results of the present study suggest that combined therapy with corticosteroids, ascorbic acid, and thiamin may be effective in reducing the severity of impaired oxygenation following esophagectomy. Although this small pilot retrospective study has limitations, this safe and inexpensive combination therapy has the potential to improve $\mathrm{P}_{\mathrm{a}} \mathrm{O}_{2} / \mathrm{F}_{\mathrm{i}} \mathrm{O}_{2}$ ratios after esophagectomy. Additional large-scale prospective studies are required to confirm these preliminary findings.

\section{Disclosure Statement}

None.

\section{References}

1) Haga Y, Beppu T, Doi K, et al. 1997;. Systemic inflammatory response syndrome and organ dysfunction following gastrointestinal surgery. Crit Care Med 1997; 25: 1994-2000.

2) Takeuchi H, Miyata H, Ozawa $\mathrm{S}$, et al. Comparison of short-term outcomes between open and minimally invasive esophagectomy for esophageal cancer using a nationwide database in Japan. Ann Surg Oncol 2017; 24: $1821-7$. 
3) Mamidanna R, Bottle A, Aylin P, et al. Short-term outcomes following open versus minimally invasive esophagectomy for cancer in England: a populationbased national study. Ann Surg 2012; 255: 197-203.

4) Boshier PR, Marczin N, Hanna GB. Pathophysiology of acute lung injury following esophagectomy. Dis Esophagus 2015; 28: 797-804.

5) Baudouin SV. Lung injury after thoracotomy. Br J Anaesth 2003; 91: 132-42.

6) Yamada T, Hisanaga M, Nakajima $Y$, et al. Serum interleukin-6, interleukin-8, hepatocyte growth factor, and nitric oxide changes during thoracic surgery. World J Surg 1998; 22: 783-90.

7) Wang ZQ, Chen LQ, Yuan Y, et al. Effects of neutrophil elastase inhibitor in patients undergoing esophagectomy: a systematic review and meta-analysis. World J Gastroenterol 2015; 21: 3720-30.

8) Weijs TJ, Dieleman JM, Ruurda JP, et al. The effect of perioperative administration of glucocorticoids on pulmonary complications after transthoracic oesophagectomy: a systematic review and meta-analysis. Eur J Anaesthesiol 2014; 31: 685-94.

9) Engelman E, Maeyens C. Effect of preoperative singledose corticosteroid administration on postoperative morbidity following esophagectomy. J Gastrointest Surg 2010; 14: 788-804.

10) Zeiher BG, Artigas A, Vincent JL, et al. Neutrophil elastase inhibition in acute lung injury: results of the STRIVE study. Crit Care Med 2004; 32: 1695-702.

11) Marik PE. Hydrocortisone, ascorbic acid and thiamine (HAT therapy) for the treatment of sepsis. Focus on ascorbic acid. Nutrients 2018; 10: 1762.

12) Barabutis N, Khangoora V, Marik PE, et al. Hydrocortisone and ascorbic acid synergistically prevent and repair lipopolysaccharide-induced pulmonary endothelial barrier dysfunction. Chest 2017; 152: 954-62.

13) Marik PE, Khangoora V, Rivera R, et al. Hydrocortisone, vitamin $\mathrm{C}$, and thiamine for the treatment of severe sepsis and septic shock: a retrospective beforeafter study. Chest 2017; 151: 1229-38.

14) Marik PE, Long A. ARDS complicating pustular psoriasis: treatment with low-dose corticosteroids, vitamin C and thiamine. BMJ Case Rep 2018; 2018: 223475.

15) Fowler AA, Syed AA, Knowlson S, et al. Phase I safety trial of intravenous ascorbic acid in patients with severe sepsis. J Transl Med 2014; 12: 32.

16) The Japan Esophageal Society. Guidelines for Diagnosis and Treatment of Carcinoma of the Esophagus, Tokyo: Kanehara Shuppan, 2017.

17) Tsukada K, Miyazaki T, Katoh H, et al. Effect of perioperative steroid therapy on the postoperative course of patients with oesophageal cancer. Dig Liver Dis 2006; 38: 240-4.
18) Marik PE. Fluid responsiveness and the six guiding principles of fluid resuscitation. Crit Care Med 2016; 44: $1920-2$.

19) Network Acute Respiratory Distress Syndrome, Brower RG, Matthay MA, et al. Ventilation with lower tidal volumes as compared with traditional tidal volumes for acute lung injury and the acute respiratory distress syndrome. N Engl J Med 2000; 342: 1301-8.

20) Barr J, Fraser GL, Puntillo K, et al. Clinical practice guidelines for the management of pain, agitation, and delirium in adult patients in the intensive care unit. Crit Care Med 2013; 41: 263-306.

21) The committee from the Japanese Society of Intensive Care Medicine, the Japanese Society of Respiratory Care Medincine and Japan Academy of Critical Care Nursing on protocol for liberation from mechanical ventilation. Japanese protocol for liberation from mechanical ventilation in critical ill adults. 2015.

22) Padayatty SJ, Sun H, Wang Y, et al. Vitamin C pharmacokinetics: implications for oral and intravenous use. Ann Intern Med 2004; 140: 533-7.

23) Schacter EN, Littner MR, Luddy P, et al. Monitoring of oxygen delivery systems in clinical practice. Crit 1980; 8: 405-9.

24) Makino H, Kunisaki C, Kosaka T, et al. Perioperative use of a neutrophil elastase inhibitor in video-assisted thoracoscopic oesophagectomy for cancer. Br J Surg 2011; 98: 975-82.

25) Fisher BJ, Seropian IM, Kraskauskas D, et al. Ascorbic acid attenuates lipopolysaccharide-induced acute lung injury. Crit Care Med 2011; 39: 1454-60.

26) Marik PE. Critical illness-related corticosteroid insufficiency. Chest 2009; 135: 181-93.

27) Marik PE, Pastores SM, Annane D, et al. Recommendations for the diagnosis and management of corticosteroid insufficiency in critically ill adult patients: consensus statements from an international task force by the American College of Critical Care Medicine. Crit Care Med 2008; 36: 1937-49.

28) Heitzer T, Just H, Münzel T. Antioxidant vitamin $C$ improves endothelial dysfunction in chronic smokers. Circulation 1996; 94: 6-9.

29) Azari O, Kheirandish R, Azizi S, et al. Protective effects of hydrocortisone, vitamin $\mathrm{C}$ and $\mathrm{E}$ alone or in combination against renal ischemia-reperfusion injury in rat. Iran J Pathol 2015; 10: 272-80.

30) National Heart, Lung, and Blood Institute Acute Respiratory Distress Syndrome (ARDS) Clinical Trials Network. Wiedemann HP, Wheeler AP, et al. Lung, and Blood Institute Acute Respiratory Distress Syndrome (ARDS) Clinical Trials Network. N Engl J Med 2006; 354: 2564-75. 